\title{
Optimization of Multibit Watermarking
}

\author{
Joceli Mayer \\ Research Laboratory on Digital Signal Processing, Federal University of Santa Catarina
}

Brazil

\section{Introduction}

This Chapter presents a Multibit Improved Spread Spectrum modulation (MISS) by properly adjusting the energy of the pseudo random sequences modulated by Code Division (CDM). We extend the one-bit spread spectrum watermarking approach proposed by Henrique S. Malvar and Dinei A. F. Florencio (2003) to multibit watermarking by using an optimization procedure to achieve the best performance possible in robustness and transparency while mitigating the cross correlations among sequences and the host interference. The proposed multibit approach also tradeoffs the resulting watermarking distortion with the host interference rejection. This Chapter extends the approach published in Mayer (2011). We describe the improved modulation method and present results to illustrate the performance.

\subsection{Spread spectrum modulation}

Spread spectrum modulation is a mature and popular approach for hiding information in a host [I. J. Cox, J. Kilian, F. T. Leighton, T. Shamoon (1997)]. The basic idea is to spread one message bit over many samples of the host data. The spreading can be achieved by modulating the host data with a sequence obtained from a pseudo-random generator, for instance.

In general and independently of the media type, the embedding in the sample domain of one antipodal bit $b$ can be achieved by additive embedding [I. J. Cox, J. Kilian, F. T. Leighton, T. Shamoon (1997)]

$$
\boldsymbol{c}=\boldsymbol{c}_{0}+\alpha b \boldsymbol{p}
$$

where $c_{0}$ is the vector representation of the $M$ samples of the host data, $\boldsymbol{p}$ is the vector representation of the spread sequence of size $M, \alpha$ is a scaling parameter, $b$ is an antipodal bit $\in\{-1,+1\}, \boldsymbol{w}=\alpha b \boldsymbol{p}$ is the embedded watermark and $\boldsymbol{c}$ is the vector representation of the resulting watermarked data of size $M$. The embedding can be applied to any document type (voice, audio, image, video, text, etc) and in any feature space (samples, linearly transformed domain using wavelet (WT), discrete cosine (DCT) or Fourier transform (FT), or another space). By spreading the information over the chosen domain, all spread spectrum modulation techniques [I. J. Cox, M. L. Miller, J. A. Bloom (2002)] can provide robustness to non-malicious attacks such as compression, filtering, histogram equalization and A/D and D/A conversions, as well as to tampering attacks [I. J. Cox, J. Kilian, F. T. Leighton, T. Shamoon (1997); I.J. Cox, M.L. Miller, A.L. Mckellips (1999); Santi P. Maity and Malay K. Kundu (2004); Z. Jane Wang, Min Wu, Hong Vicky Zhao, Wade Trappe, K. J. Ray Liu (2005)]. Spread spectrum watermarking can be made robust to geometric operations such 
as rotation, cropping and scaling by employing a pre-processing tailored to the specific attack [I. J. Cox, J. Kilian, F. T. Leighton, T. Shamoon (1997); Yanmei Fang, Jiwu Huang, Shaoquan $\mathrm{Wu}(2004)]$. There has been a great deal of effort towards the generation and shaping of sequences $\boldsymbol{p}$, that can improve the performance of the watermarking system [Mauro Barni and Franco Bartolini (2004)]. Reduction of the perceptual impact can be achieved by exploiting the human perceptual masking in either the frequency or the sample domain [E.J. (1999); Joceli Mayer and José C. M. Bermudez (2005); Joseph J.K.Ó. Ruanaidh and Gabriella Csurka (1999); Martin Kutter and Stefan Winkler (2002); Santi P. Maity and Malay K. Kundu (2004)]. The system performance can be measured by estimating the probability of detection considering channel distortions and/or malicious attacks, by evaluating the distortion resulting from the insertion of the watermark signal into the host data, by determining the computational resource requirements (speed of detection, required memory), or by other practical constraint imposed by the application. Some recent results [Henrique S. Malvar and Dinei A. F. Florencio (2003); M. Barni, N. Memon, T. Kalker, P. Moulin, I.J. Cox, M.L. Miller, J.J. Eggers, F. Bartolini, F. Pérez-González (2003); Perez-gonzalez \& Pun (2004)] indicate that informed embedding spread spectrum watermarking can provide a competitive performance when compared to techniques based on the dirty-paper approach [Moulin \& Koetter (2005)], especially in practical situations when scaling attacks are considered and the noise and the host signals cannot be properly modeled by Additive White Gaussian Noise (AWGN) sequences.

Some simple and popular schemes focus on combining classical 1-bit spread spectrum modulation with an informed embedding strategy. An "erase and write" strategy has been initially proposed in I. J. Cox, M. L. Miller, J. A. Bloom (2002) and named "Peaking DS" (PEAK) in Delhumeau et al. (2003). It consists of pre-canceling the host interference before embedding:

$$
\boldsymbol{w}=\alpha \boldsymbol{p}-\frac{<\boldsymbol{p}, \boldsymbol{c}_{0}>}{<\boldsymbol{p}, \boldsymbol{p}>} \boldsymbol{p}
$$

where $\left\langle\boldsymbol{p}, \boldsymbol{c}_{0}\right\rangle$ stands for the inner product of $\boldsymbol{p}$ and $\boldsymbol{c}_{0}$. Improved Spread Spectrum (ISS) is an extension to PEAK that maximizes the robustness to an AWGN attack of known power $\sigma_{\eta}^{2}$, at constant distortion [Henrique S. Malvar and Dinei A. F. Florencio (2003)]. A compromise is made between the power of the watermark portion devoted to host-interference cancellation and its information-carrying portion, the latter essential for robustness to attacks.

\subsection{Multibit spread spectrum watermarking}

Many applications require a higher payload watermarking [Mauro Barni and Franco Bartolini (2004)]. In these cases, one bit watermarking can be extended to convey more bits of information. By using a codebook of sequences, which should be known by both the encoder and the decoder, a multibit watermark can be designed to convey $N$ bits of information. In most cases, only a secret key $K$ is needed to recreate the sequences at decoder. This key is usually shared by the sender and receiver using a secure protocol and neither the host image or the sequences are required to be known by the receiver in the blind decoding watermarking. Three modulation approaches are possible: basic message coding, Time Division Multiplexing (TDM) and Code Division Multiplexing (CDM). Basic message coding [I. J. Cox, M. L. Miller, J. A. Bloom (2002)] requires a unique sequence $\boldsymbol{w}_{i}$ for each $n$-bit message $m_{i}$, resulting in a codebook with $2^{N}$ possible messages. Issues associated with this approach include computational complexity for generating and, in some cases, storing the codebook. Moreover, the detection process requires a search for a sequence in a space of $2^{N}$ vectors of dimension 
$M$. Depending on the required payload ( $N$ bits), this multibit approach may require a huge computational complexity [Mauro Barni and Franco Bartolini (2004)]. The search complexity can be considerably reduced by structuring the sequences into a binary tree [Wade Trappe, Min Wu, Jane Wang, K. J. Ray Liu (2003)], which requires the storage of the sequences. On the other hand, using a binary tree may increase both the storage requirements and the probability of detecting the wrong message when the sequences are not perfectly orthogonal to each other. For instance, consider that a pseudo random generator (PN) based on a secret key $K$ is used to generate the codebook. For security, only the intended decoder agent should detain the knowledge of the sequence $\boldsymbol{p}$ or of the key $K$ that generated the sequence. Assume that the Lehmer generator [? is used with $L=2^{31}-1$, where $L$ is the period of the generator. To embed messages into images with $S^{2}=512^{2}=2^{18}$ pixels, the generator can provide, without overlapping sequences, a maximum of $L / S^{2}=2^{31} / 2^{18}=2^{13}$ sequences and messages. This means that only 13 bits can be embedded in the host data. By allowing overlapping, the generator can provide $2^{31}-1$ different sequences, resulting in messages of at most 30 bits.

Alternatives based on the spread spectrum approach aimed at reducing the complexity of multibit watermarking include the use of message multiplexing such as TDM and CDM [Mauro Barni and Franco Bartolini (2004)]. The computational complexity required for detection is linear in $N(O(N)$ for embedding $N$ bits), whereas the basic message coding and orthogonal modulation require an exponential $\left(O\left(2^{N}\right)\right)$ number of detections [Z. Jane Wang, Min Wu, Hong Vicky Zhao, Wade Trappe, K. J. Ray Liu (2005)]. In TDM, the host signal is divided into $N$ subsets of size $M / N$. Then 1-bit spread spectrum watermark embedding is performed on each subset. A combination of these multiplexing techniques can provide a desired tradeoff for a given application. For instance, a mixed embedding based on TDM and basic message coding is employed in [Min Wu and Bede Liu (2003)] to mitigate the computational complexity associated with the basic message coding approach. Random sample shuffling is often necessary for TDM to deal with the uneven capacity of each signal segment (non-stationary signals) [Min Wu and Bede Liu (2003)]. CDM multiplexing is discussed next.

\subsection{CDM spread spectrum watermarking}

In general, a watermarking technique employing CDM embeds $N$ bits into the host data (or in a feature space) $\boldsymbol{c}_{0}$, resulting in the watermarked signal

$$
\boldsymbol{c}=\boldsymbol{c}_{0}+\boldsymbol{w}=\boldsymbol{c}_{0}+\alpha \sum_{j=1}^{N} b_{j} \boldsymbol{p}_{j}
$$

The host data vector $\boldsymbol{c}_{0}$ (samples, coefficients of a transformed domain or host features) and the watermarked signal $c$ can represent speech, image or video signals where their samples are organized as a vector of dimension $M$. In this case, the watermark is $\boldsymbol{w}$. The multi-bit message vector $\boldsymbol{b}$ is composed by $N$ antipodal bits $b_{j}, j=1, \ldots, N$. The scaling factor $\alpha$ controls the energy of the resulting multibit watermark $\boldsymbol{w}$. Each M-dimensional vector $\boldsymbol{p}_{j}, j=1, \ldots, N$, contains a spread sequence (the $j$-th spread sequence) with $M$ samples, usually obtained using a pseudo random generator. Vectors $\boldsymbol{p}_{j}$ can be shaped applying a masking vector $\boldsymbol{x}$, resulting in an adjusted vector $\boldsymbol{p}_{j} * \boldsymbol{x}$, where $*$ represents element by element vector multiplication. This perceptual masking can be designed to achieve a more transparent embedding according to a perceptual-based criterion. 
Techniques with different performances and tuned to specific applications can be derived using different combinations of spreading sequences, masking operators, weighting factor evaluation techniques and embedding domains [Henrique S. Malvar and Dinei A. F. Florencio (2003); Perez-gonzalez \& Pun (2004); Santi P. Maity and Malay K. Kundu (2004); Yanmei Fang, Jiwu Huang, Shaoquan $\mathrm{Wu}(2004)]$. When the sequences $\boldsymbol{p}_{j}$ are designed such that they do not overlap with each other, the CDM becomes a TDM multiplexing. This approach was used in Martin Kutter (1999) to deal with ISI. Thus, TDM can be seen as a special case of CDM [I. J. Cox, M. L. Miller, J. A. Bloom (2002)].

CDM has been widely employed for watermark embedding in both the sample and transformed domains [Joseph J.K.Ó. Ruanaidh and Gabriella Csurka (1999); Santi P. Maity and Malay K. Kundu (2004); Yongqing Xin and Miroslaw Pawlak (2004)]. On the other hand, the perceptual impact of the CDM embedding on the fidelity increases with the number $N$ of bits embedded when the spreading sequences overlap with each other, generating intersymbol interference (ISI) [Martin Kutter (1999)]. This impact can be mitigated by using M-ary modulation [Martin Kutter (1999)], which, on the other hand, increases the detection complexity and precludes the error performance to degrade graciously [Mauro Barni and Franco Bartolini (2004)]. The cross-correlation effects can be mitigated by designing spreading sequences using pseudo random generators followed by the Gram-Schmidt orthogonalization, by using orthogonal sequences created from a Walsh-Hadamard basis [J. Mayer, A. V. Silverio, J. C. M. Bermudez (2002); Santi P. Maity and Malay K. Kundu (2004)] or through other orthogonalization techniques. Performance improvement can be also achieved by employing Wiener pre-filtering to mitigate host correlation [Hernández \& Pérez-González (1999)]. Many approaches in the literature propose to embed the watermark into reduced length regions of the data. These include many space-domain block-based embedding schemes [Borges \& Mayer (2006); Paulo V. K. Borges, Joceli Mayer (2005)] and frequency-domain schemes that segment the watermark representation using, for instance, the DCT with $8 \times 8$ blocks or wavelet decompositions. Unfortunately, however, the pseudo random generators used in practice generate highly cross-correlated short sequences. The degrading effect of cross-correlated sequences on the detector performance becomes especially important in applications that require small bit error rates (BER). The improvements proposed in this Chapter for CDM address the low BER case for highly cross-correlated sequences.

\section{Improved CDM}

The traditional CDM watermarking approach in (3) scales the watermark energy by a factor $\alpha^{2}$. This approach is inefficient because it relies on a single factor $\alpha$ to adjust the energy of all sequences. Given a fixed gain factor $\alpha$ designed to minimize some cost function, many patterns $\boldsymbol{p}_{j}$ can be introduced with more (or less) energy than the minimum necessary to satisfy the robustness or the fidelity constraints. Moreover, the patterns need to be designed considering the interferences caused by the host image, by the cross-correlation among sequences and by the perceptual shaping mask $\boldsymbol{x}$. Otherwise, the designed patterns will provide sub-optimal embedding, resulting in losses in transparency and robustness. An analysis of alternative pattern generating methods can be found in J. Mayer, A. V. Silverio, J. C. M. Bermudez (2002). Perceptual masking might affect the orthogonality of the spreading sequences and compromise the detector performance [Joceli Mayer and José C. M. Bermudez (2005)]. In some schemes perceptual masking is not employed or even implemented in an 
alternative way. The proposed approach also addresses this interference by considering the masking effects on the ISI. We address the watermark embedding issue by allowing a different gain factor $\alpha_{j}$ for each pattern [Joceli Mayer, Rafael Araujo da Silva (2004)]. Thus, we propose to embed $N$ bits into the host data by using

$$
\boldsymbol{c}=\boldsymbol{c}_{0}+\sum_{j=1}^{N} \alpha_{j} b_{j} \boldsymbol{p}_{j} * \boldsymbol{x}
$$

In the following we assume that the spreading sequence vectors $\boldsymbol{p}_{j}$ are zero-average. We also assume that the additive transmission channel noise $\boldsymbol{\eta}$ is zero-mean, statistically independent and identically distributed (i.i.d.), with an even probability density function (pdf), but not necessarily Gaussian. We also define a decision variable $d_{i}$, relative to bit $b_{i}$, which is computed at the detector using linear correlation of the spreading sequence with the received watermarked signal:

$$
\begin{array}{r}
d_{i}=\left\langle\boldsymbol{p}_{i}, \boldsymbol{c}+\boldsymbol{\eta}\right\rangle \\
d_{i}=\left\langle\boldsymbol{p}_{i}, \boldsymbol{c}_{0}\right\rangle+\left\langle\boldsymbol{p}_{i}, \sum_{j=1}^{N} \alpha_{j} b_{j} \boldsymbol{p}_{j} * \boldsymbol{x}\right\rangle+\left\langle\boldsymbol{p}_{i}, \boldsymbol{\eta}\right\rangle \\
d_{i}=R_{i}^{\boldsymbol{c}_{0}}+R_{i}^{\eta}+\left\langle\boldsymbol{p}_{i}, \sum_{j=1}^{N} \alpha_{j} b_{j} \boldsymbol{p}_{j} * \boldsymbol{x}\right\rangle \\
=R_{i}^{\boldsymbol{c}_{0}}+R_{i}^{\eta}+\sum_{j=1}^{N} \alpha_{j} R_{i j}
\end{array}
$$

where $R_{i}^{\boldsymbol{c}_{0}}=\left\langle\boldsymbol{p}_{i}, \boldsymbol{c}_{0}\right\rangle, R_{i}^{\eta}=\left\langle\boldsymbol{p}_{i}, \boldsymbol{\eta}\right\rangle$ and $R_{i j}=b_{j}\left\langle\boldsymbol{p}_{i}, \boldsymbol{p}_{j} * \boldsymbol{x}\right\rangle$ are, respectively, the correlation of $\boldsymbol{p}_{i}$ with the host image, the correlation of $\boldsymbol{p}_{i}$ with the noise and the cross-correlation between $\boldsymbol{p}_{i}$ and the pattern $\boldsymbol{p}_{j}$ multiplied, element by element, by the mask $\boldsymbol{x}$. Notice that we employ linear correlation for detection, as is usually the case in most practical watermarking systems. However, the linear correlation is optimal only when the involved signals are Gaussian distributed. A discussion about the optimality of the linear detector is given in Mauro Barni and Franco Bartolini (2004).

For the noiseless case, $R_{i}^{\eta}=0$ and we can guarantee a specified detection level $d_{i}=\beta b_{i}$, for $i=1, \cdots, N$, by solving (5) for the gain factor vector $\boldsymbol{\alpha}=\left[\alpha_{1}, \cdots, \alpha_{N}\right]^{T}$ :

$$
\left[\begin{array}{cccc}
R_{11} & R_{12} & \cdots & R_{1 N} \\
R_{21} & R_{22} & & \vdots \\
\vdots & & \ddots & \\
R_{N 1} & \cdots & & R_{N N}
\end{array}\right] \cdot\left[\begin{array}{c}
\alpha_{1} \\
\alpha_{2} \\
\vdots \\
\alpha_{N}
\end{array}\right]=\left[\begin{array}{c}
\beta \cdot b_{1}-R_{1}^{c_{0}} \\
\beta \cdot b_{2}-R_{2}^{c_{0}} \\
\vdots \\
\beta \cdot b_{N}-R_{N}^{c_{0}}
\end{array}\right]
$$

This approach simultaneously takes into account the interferences from the host image, patterns and shaping mask in order to enforce that $d_{i}=\beta b_{i}$ for all bits. Notice that $R_{i j}<<R_{i i}$ (disregarding the mask $x$ ) for practical pseudo-random generators, assuring that the matrix in Eq. (6) is almost diagonal and has rank $N$ so that there exists a solution for $\boldsymbol{\alpha}$. Considering an additive noise with known power, the parameter $\beta$ can be determined to compensate for the effect of the $R_{i}^{\eta}$ correlations, as discussed in Joceli Mayer and José C. M. Bermudez (2005). 


\section{Extending PEAK and ISS schemes}

The proposed approach extends the single-bit PEAK Delhumeau et al. (2003) strategy to multibit and also tradeoffs the host interference rejection with the resulting watermark energy. The PEAK strategy is a special case of ISS using $\lambda=1$. As reported by Henrique S. Malvar and Dinei A. F. Florencio (2003), $\lambda=1$ usually provides a good but not optimal performance for the single-bit case. In this section, we investigate the extension of ISS to multibit by introducing the $\lambda$ factor. In our development, the factor $\lambda$ represents the amount of host interference being cancelled, where $\lambda=1$ indicates complete cancelation.

The proposed approach extends (6) proposed in Joceli Mayer and José C. M. Bermudez (2005) by introducing the parameter $\lambda$, similarly as the one-bit ISS scheme, to tradeoff host rejection with watermark energy. For the noiseless case, $R_{i}^{\eta}=0$, the resulting detection level $d_{i}=$ $\beta b_{i}+(1-\lambda) R_{i}^{c_{0}}$ is affected by the residual of host correlation, for $i=1, N$. This clearly indicates that the constant robustness property [Joceli Mayer and José C. M. Bermudez (2005)] cannot be achieved when $\lambda \neq 1$. For a given pair $\lambda$ and $\beta$, this detection level can be enforced by solving the following system for $\alpha$ :

$$
\left[\begin{array}{lll}
R_{11} & R_{12} & R_{1 N} \\
R_{21} & R_{22} & \\
R_{N 1} & & R_{N N}
\end{array}\right] \cdot\left[\begin{array}{l}
\alpha_{1} \\
\alpha_{2} \\
\alpha_{N}
\end{array}\right]=\left[\begin{array}{l}
\beta b_{1}+(1-\lambda) R_{1}^{c_{0}} \\
\beta b_{2}+(1-\lambda) R_{2}^{c_{0}} \\
\beta b_{N}+(1-\lambda) R_{N}^{c_{0}}
\end{array}\right]
$$

The resulting detection level, considering an additive channel noise, is given by:

$$
d_{i}=\beta b_{i}+(1-\lambda) R_{i}^{c_{0}}+R_{i}^{\eta}
$$

and the error probability can be different for each bit:

$$
P E_{i}=\operatorname{Pr}\left(R_{i}^{\eta}>\beta-(1-\lambda) R_{i}^{c_{0}}, b_{i}=-1\right)+\operatorname{Pr}\left(R_{i}^{\eta}<-\beta-(1-\lambda) R_{i}^{c_{0}}, b_{i}=1\right)
$$

Thus, our average bit error probability is given by:

$$
P_{e M}=\frac{1}{N} \sum_{i=1}^{N} P E_{i}
$$

After solving (7), the resulting introduced distortion will be:

$$
s_{W}^{2}=\left(\sum_{i=1}^{N} \alpha_{i} \beta \boldsymbol{p}_{i}\right)^{T}\left(\sum_{i=1}^{N} \alpha_{i} \beta \boldsymbol{p}_{i}\right)
$$

We employ an optimization approach to find the proper $\beta$ and $\lambda$ parameters, as follows. For a given desired maximum average probability, $P M$, we compute the $J * K$ points from $P_{e M}\left(\beta_{j}, \lambda_{k}\right)$ and $s_{W}^{2}\left(\beta_{j}, \lambda_{k}\right)$ for each pair $\left(\beta_{j}, \lambda_{k}\right)$. The search range is defined by $\beta_{j}=\beta_{\text {min }}+$ $j *\left(\beta_{\max }-\beta_{\text {min }}\right) / J, j=0, \cdots, J-1$ and $\lambda_{k}=\lambda_{\min }+k *\left(\lambda_{\max }-\lambda_{\min }\right) / K, k=0, \cdots, K-1$ , where $J$ and $K$ are the chosen number of points and $<\beta_{\min }, \beta_{\max }, \lambda_{\min }, \lambda_{\text {max }}>$ are positive values chosen tipically as $<0,5,0,1\rangle$. For each pair $\left(\beta_{j}, \lambda_{k}\right)$ it is required to compute the resulting $P_{e M}\left(\beta_{j}, \lambda_{k}\right)$, and if it is less than $P M$, the value $s_{W}^{2}\left(\beta_{j}, \lambda_{k}\right)$ is also required by first solving (7). The pair that results in the smallest $s_{W}^{2}\left(\beta_{j}, \lambda_{k}\right)$, restricted to $P_{e M}\left(\beta_{j}, \lambda_{k}\right)<P M$, will be chosen. It is possible that no pair satisfies the restriction, in this case $P M$ needs to be increased. The approach requires, in the worst case, $J * K$ computations of $P_{e M},(7)$ and of resulting $s_{W}^{2}$. These cost functions, $s_{W}^{2}\left(\beta_{j}, \lambda_{k}\right), P_{e M}\left(\beta_{j}, \lambda_{k}\right)$ are illustrated at Fig. 1. 


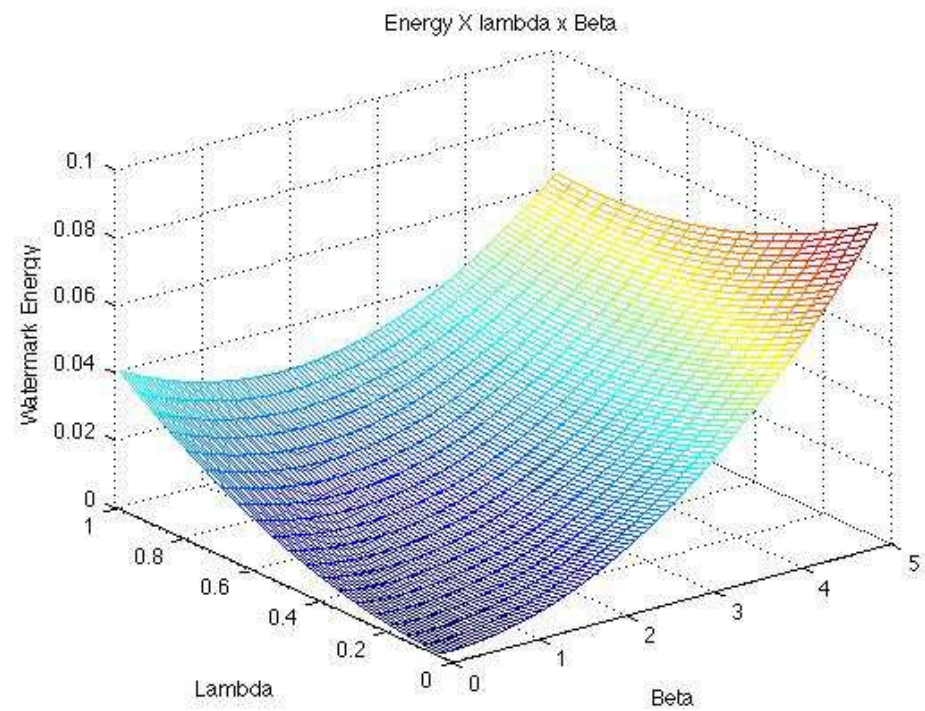

(a)

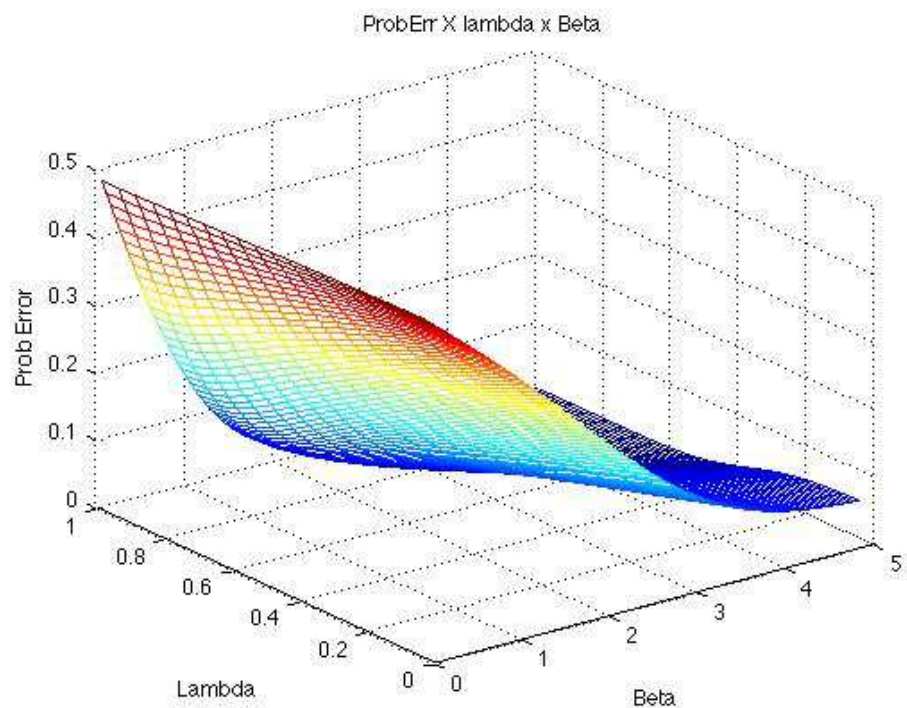

(b)

Fig. 1. (a) Watermark energy versus parameters $\beta$ and $\lambda$. (b) Probability of error versus parameters $\beta$ and $\lambda$. Optimized performance: very transparent $\left(s_{W}^{2}=0.0587\right)$ and the Monte Carlo simulation validate the specified probability of error $(\mathrm{Pe} \leq 1 \mathrm{E}-6)$ resulting in the measured of $P_{e M}=7.93 E-7$. 


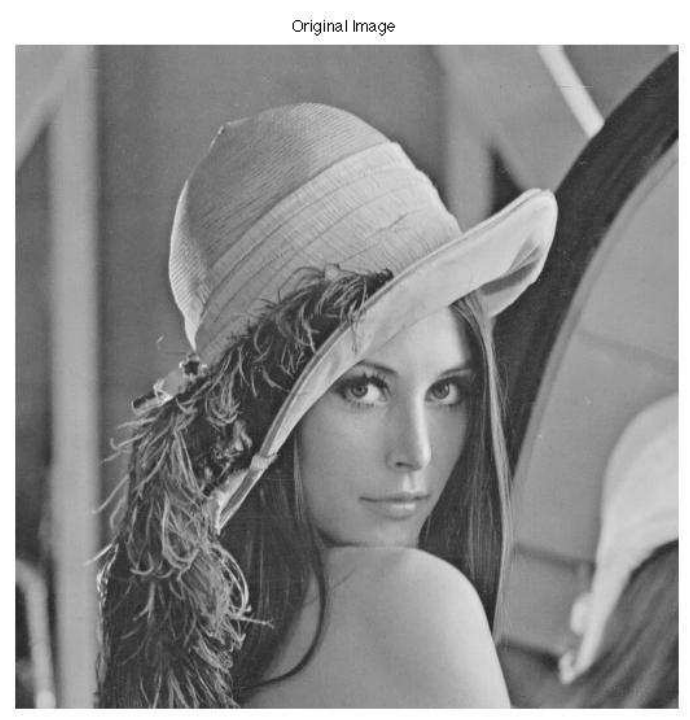

(a)

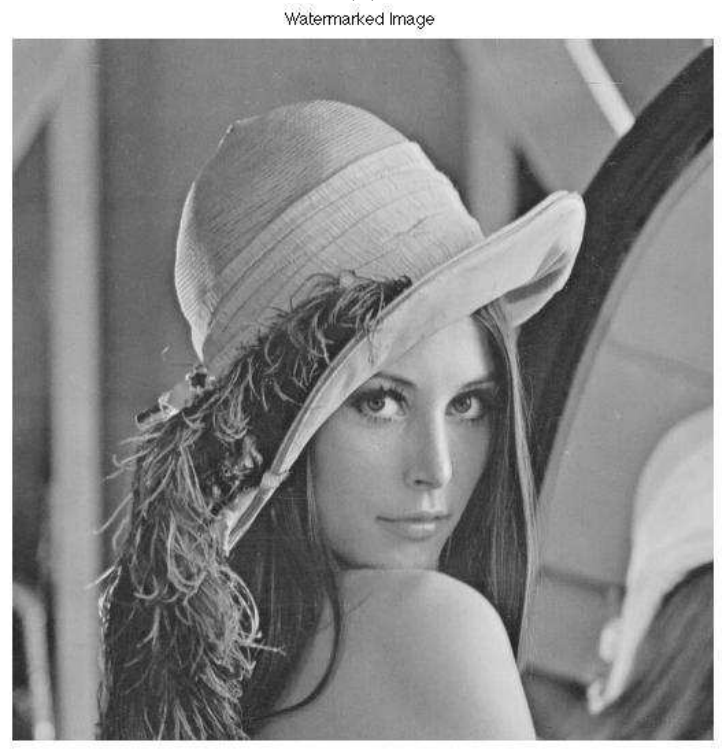

(b)

Fig. 2. (a) Original Image, (b) Watermarked Image (very low perceptual impact, $s_{W}^{2}=0.0587$, embedded with 10 bits using the MISS). 
Scaled Difference Between Original and Watermarked Image

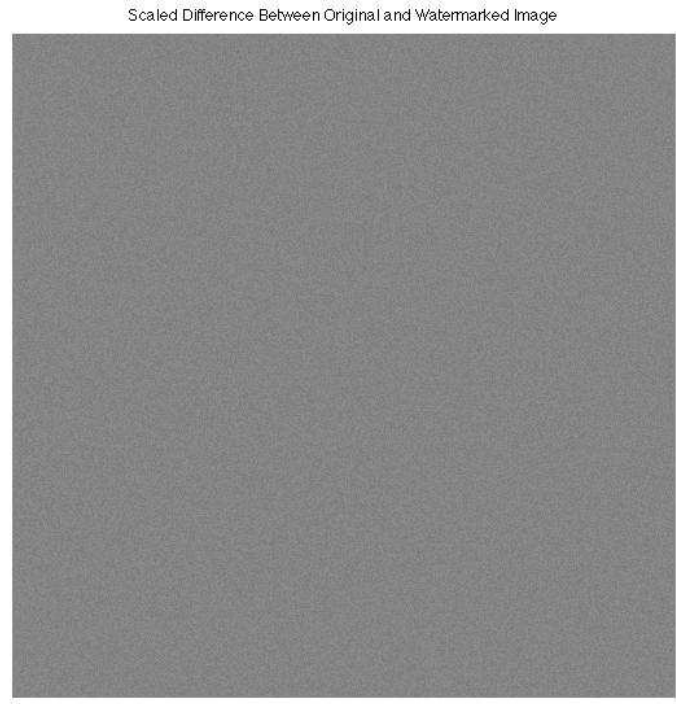

(a)

Watermarked with channel distortions

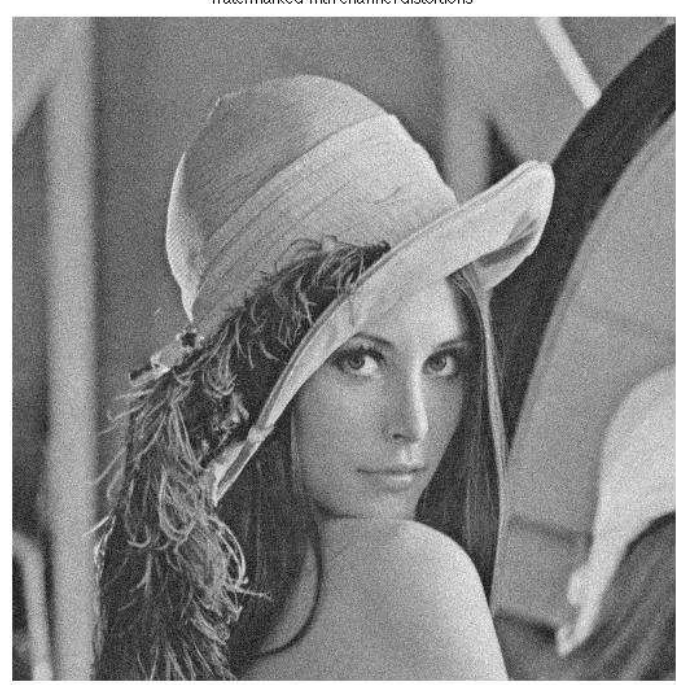

(b)

Fig. 3. (a) Difference between the original and the watermarked Image (scaled by 10 and added 128), (b) Watermarked Image attacked with AWGN noise $\sim N(0,10)$. Very robust to AWGN, $P_{\text {error }} \leq 1 E-6$. 


\section{Experiments}

The figures 2 and 3 show the performance using the proposed optimization, presenting high transparency and very low probability of error for the AWGN attack. Perceptual masking is not implemented in this example designed to observe the benefits of mitigating host interference and cross correlation among patterns. By contrasting to the traditional multibit $\mathrm{CDM}$, in (3), on a Monte Carlo experiment with 1000 trials, AWGN noise $\sim \mathrm{N}(0,10)$, and adjusting the schemes for the same watermark energy in all tests, the resulting average error probability $\left(P_{e M}\right)$ and its deviation for CDM was $P_{e M-C D M}=0.0425 \pm 0.0478$ while for the proposed Multibit Improved Spread Spectrum, we found $P_{e M-M I S S}=0.019 \pm 0.00047$. The MISS approach provides considerably smaller probability of error in both average and deviation when compared to the traditional CDM.

Note that the MISS is based on Spread Spectrum (SS) modulation and presents similar robustness to compression and filtering attacks as other related SS techniques [Delhumeau et al. (2003); Henrique S. Malvar and Dinei A. F. Florencio (2003)]. Particularly, the proposed approach is designed to extend the ISS technique to multibit embedding. Thus, it is expected from the MISS approach the same performance achieved by ISS approach for 1-bit embedding. MISS provides a superior multibit embedding than the PEAK approach [Delhumeau et al. (2003)] as it finds the best values for $\beta$ and $\lambda$ at the multibit embedding.

\section{Conclusions}

We presented an extension of the ISS and PEAK algorithms to multibit spread spectrum. The proposed scheme estimates the probability of error for AWGN and the expected distortion for each combination of parameters $\lambda$ and $\beta$. The scheme outperforms previous proposed multibit CDM spread spectrum modulation [Joceli Mayer and José C. M. Bermudez (2005)] as it finds the least energy necessary for the watermark given a target probability of error. The proposed modulation approach, coined as MISS, is applicable to image, audio, speech and video media. Further improvement can be achieved by extending the proposed optimization by using one parameter $\lambda_{i}$ per message bit.

\section{References}

Borges, P. V. K. \& Mayer, J. (2006). Analysis of position based watermarking, Pattern Analysis $\mathcal{E}$ Applications, Springer London 9(1): 70-82.

Delhumeau, J., Furon, T., Hurley, N. \& Silvestre, G. (2003). Improved polynomial detectors for side-informed watermarking, Proc. SPIE .

E.J., W. R. P. C. D. (1999). Perceptual watermarks for digital images and video, Proceedings of the IEEE 87(7): 1108-1126.

Henrique S. Malvar and Dinei A. F. Florencio (2003). Improved spread spectrum: A new modulation technique for robust watermarking, IEEE Trans. on Signal Processing 51(4): 898-905.

Hernández, J. \& Pérez-González, F. (1999). Statistical analysis of watermarking schemes for copyright protection of images, IEEE Proc., Special Issue on Identification and Protection of Multimedia Information 87(7): 1142-1166. 
I. J. Cox, J. Kilian, F. T. Leighton, T. Shamoon (1997). Secure spread spectrum watermarking for multimedia, IEEE Transactions on Image Processing 6(12): 1673-1687.

I. J. Cox, M. L. Miller, J. A. Bloom (2002). Digital Watermarking, Morgan Kaufmann.

I.J. Cox, M.L. Miller, A.L. Mckellips (1999). Watermarking as communications with side information, Proceedings of the IEEE 87(7): 1127-1141.

J. Mayer, A. V. Silverio, J. C. M. Bermudez (2002). On the design of pattern sequences for spread spectrum image watermarking, Int. Telecommunications Symposium, ITS .

Joceli Mayer and José C. M. Bermudez (2005). Multi-bit informed embedding watermarking with constant robustness, IEEE Intl. Conf. on Image Processing, ICIP 1: 804-821.

Joceli Mayer, Rafael Araujo da Silva (2004). Efficient informed embedding of multi-bit watermark, IEEE International Conference on Acoustics, Speech, and Signal Processing, ICASSP, Vol. 1, pp. 389-392.

Joseph J.K.Ó. Ruanaidh and Gabriella Csurka (1999). A bayesian approach to spread spectrum watermark detection and secure copyright protection for digital image libraries, IEEE Comp. Society Conf. on Comp. Vision and Pattern Recognition, pp. 207-212.

M. Barni, N. Memon, T. Kalker, P. Moulin, I.J. Cox, M.L. Miller, J.J. Eggers, F. Bartolini, F. Pérez-González (2003). Signal processing forum: What is the future for watermarking ? (part 2), IEEE Signal Processing Magazine 20(6): 53-57.

Martin Kutter (1999). Performance improvement of spread spectrum based image watermarking schemes through m-ary modulation, Lecture Notes in Computer Science, Springer Verlag 1768: 238-250.

Martin Kutter and Stefan Winkler (2002). A vision-based masking model for spread-spectrum image watermarking, IEEE Trans. on Image Processing 11(1): 16-25.

Mauro Barni and Franco Bartolini (2004). Watermarking Systems Engineering - Enabling Digital Assets Security and Other Applications, Marcel Dekker.

Mayer, J. (2011). Improved spread spectrum multibit watermarking, IEEE Intl. Workshop on Information Forensics and Security - WIFS'11 .

Min Wu and Bede Liu (2003). Data hiding in image and video: Part i-fundamental issues and solutions, IEEE Trans. on Image Processing 12(6): 685-695.

Moulin, P. \& Koetter, R. (2005). Data-hiding codes, Proceedings IEEE 93(12): 2083-2127.

Paulo V. K. Borges, Joceli Mayer (2005). Informed positional embedding for multi-bit watermarking, IEEE Intl. Conf. on Acoustics, Speech, and Signal Processing, ICASSP, Vol. 2, pp. 809-812.

Perez-gonzalez, O. K. S. V. F. D. F. \& Pun, T. (2004). Spread spectrum watermarking for real images: is everything so hopeless?, Proceedings of 12th European Signal Processing Conference, EUSIPCO.

Santi P. Maity and Malay K. Kundu (2004). A blind CDMA image watermarking scheme in wavelet domain, Intl. Conf. on Image Processing, ICIP, pp. 2633-2636.

Wade Trappe, Min Wu, Jane Wang, K. J. Ray Liu (2003). Anti-collusion fingerprinting for multimedia, IEEE Trans. on Signal Processing 51(4): 1069-1087.

Yanmei Fang, Jiwu Huang, Shaoquan Wu (2004). CDMA-based watermarking resisting to cropping, Proc. of the 2004 Intl. Symposium on Circuits and Systems, ISCAS, Vol. 2, pp. 25-28.

Yongqing Xin and Miroslaw Pawlak (2004). Multibit data hiding based on cdma, Canadian Conf. on Electrical and Computer Engineering, Vol. 2, pp. 935-938. 
Z. Jane Wang, Min Wu, Hong Vicky Zhao, Wade Trappe, K. J. Ray Liu (2005). Anti-collusion forensics of multimedia fingerprinting using orthogonal modulation, IEEE Trans. on Image Processing 14(6): 804-821. 


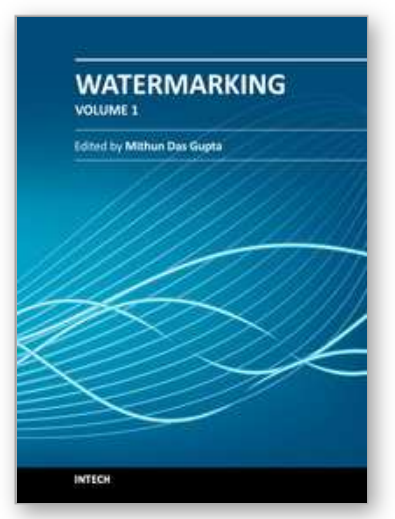

\author{
Watermarking - Volume 1 \\ Edited by Dr. Mithun Das Gupta
}

ISBN 978-953-51-0618-0

Hard cover, 204 pages

Publisher InTech

Published online 16, May, 2012

Published in print edition May, 2012

This collection of books brings some of the latest developments in the field of watermarking. Researchers from varied background and expertise propose a remarkable collection of chapters to render this work an important piece of scientific research. The chapters deal with a gamut of fields where watermarking can be used to encode copyright information. The work also presents a wide array of algorithms ranging from intelligent bit replacement to more traditional methods like ICA. The current work is split into two books. Book one is more traditional in its approach dealing mostly with image watermarking applications. Book two deals with audio watermarking and describes an array of chapters on performance analysis of algorithms.

\title{
How to reference
}

In order to correctly reference this scholarly work, feel free to copy and paste the following:

Joceli Mayer (2012). Optimization of Multibit Watermarking, Watermarking - Volume 1, Dr. Mithun Das Gupta (Ed.), ISBN: 978-953-51-0618-0, InTech, Available from: http://www.intechopen.com/books/watermarkingvolume-1/optimization-of-multibit-watermarking

\section{INTECH}

open science | open minds

\section{InTech Europe}

University Campus STeP Ri

Slavka Krautzeka 83/A

51000 Rijeka, Croatia

Phone: +385 (51) 770447

Fax: +385 (51) 686166

www.intechopen.com

\section{InTech China}

Unit 405, Office Block, Hotel Equatorial Shanghai

No.65, Yan An Road (West), Shanghai, 200040, China

中国上海市延安西路65号上海国际贵都大饭店办公楼 405 单元

Phone: +86-21-62489820

Fax: +86-21-62489821 
(C) 2012 The Author(s). Licensee IntechOpen. This is an open access article distributed under the terms of the Creative Commons Attribution 3.0 License, which permits unrestricted use, distribution, and reproduction in any medium, provided the original work is properly cited. 